\title{
Physicochemical properties of $v$ lhA promoters in Mycoplasma gallisepticum and their possible regulatory role
}

\author{
M. Orlov ${ }^{*}$, I. Garanina ${ }^{2}$, A. Ryasik ${ }^{1}$, E. Zykova ${ }^{1}$, G. Fisunov ${ }^{2}$, A. Sorokin ${ }^{1}$ \\ ${ }^{1}$ Institute of Cell Biophysics SB RAS, Pushchino, Russia \\ ${ }^{2}$ Federal Research and Clinical Center of Physical-Chemical Medicine of Federal Medical Biological \\ Agency, Moscow, Russia \\ *e-mail: orlovmikhailanat@gmail.com
}

Key words: mycoplasma, promoter, SIDD

Motivation and Aim: Mycoplasma gallisepticum is the pathogen affecting respiratory tract in poultry, thus causing significant economic losses to the agricultural industry. The intracellular parasite has a minimalistic genome with a reduced repertoire of transcription factors. Consequently, it is considered unable to regulate transcription by conventional mechanisms yet capable of adjusting gene expression in response to external stimuli in a highly dynamic manner with multiple regulators involved. Of particular interest here are phase variation antigens encoded by members of the variable lipoprotein and hemagglutinin $(v \operatorname{lh} A)$ gene family that to be involved in bacteria-host interaction and pathogenesis [1]. Here we report research on M. gallisepticum promoter regions physics considering its possible role in regulatory mechanisms.

Methods and Algorithms: For promoters from various M. gallisepticum strains (543vlhA promoters and 370 others) primary structure as well as several physicochemical and structural properties were assessed. This includes sequence logos and sliding window GC-content analysis as well as electrostatic potential, bendability, Stress-Induced Duplex Destabilization (SIDD), and open states activation energy profiles evaluation.

Results: Sequence analysis of $v l h A$ promoters has confirmed the presence of well-established GAA-repeats downstream of transcription start site and overall highly similar sequence context spanning for approximately $200 \mathrm{nt}$ around transcription start site. Among physical characteristics calculated for the promoters electrostatic potential and bendability profiles demonstrated flat regions associated with GAA-repeats, and open state activation energy profiles showed a pronounced slope downstream of transcription start site. The former might be involved in specific DNA-protein interactions, particularly, with a putative regulatory protein [2], while the latter could facilitate transcription bubble formation.

Conclusion: Reported physicochemical and structural properties of $v$ lhA promoters in M. gallisepticum suggests their possible connection with the unclear regulatory mechanisms underlying phase variation of surface antigens. Here we highlight certain characteristic features of $v l h A$ promoters physical properties. As expression regulation in the bacteria differs significantly from others, its alternative mechanisms including various DNA physicochemical properties involvement are to be furtherly considered.

\section{References}

1. Mazin P.V. et al. (2014) Transcriptome analysis reveals novel regulatory mechanisms in a genomereduced bacterium. Nucleic Acids Res. 42:13254-13268. DOI 10.1093/nar/gku976.

2. Liu L., Dybvig K., Panangala V.S., van Santen V.L., French C.T. (2000) GAA trinucleotide repeat region regulates M9/pMGA gene expression in Mycoplasma gallisepticum. Infect Immun. 68:871-876. DOI 10.1128/IAI.68.2.871-876.2000. 